\title{
Parametric study of non-periodic and hybrid auxetic bending-active gridshells
}

\section{$\operatorname{AUTHOR}(\mathrm{S})$ :}

Sakai, Yusuke; Ohsaki, Makoto

\section{CITATION:}

Sakai, Yusuke ... [et al]. Parametric study of non-periodic and hybrid auxetic bendingactive gridshells. Journal of the International Association for Shell and Spatial Structures 2020, 61(4): 275-284

\section{ISSUE DATE:}

2020-12

URL:

http://hdl.handle.net/2433/267866

\section{RIGHT:}

Copyright (c 2020 by Yusuke Sakai and Makoto Ohsaki.; Published by the International Association for Shell and Spatial Structures (IASS) with permission. 


\title{
PARAMETRIC STUDY OF NON-PERIODIC AND HYBRID AUXETIC BENDING-ACTIVE GRIDSHELLS
}

\author{
Yusuke SAKAI ${ }^{1}$ and Makoto OHSAKI ${ }^{2}$ \\ ${ }^{1}$ M.Eng., Dept. of Architecture and Architectural Engineering, Kyoto University \\ sakai.yusuke.52v@st.kyoto-u.ac.jp \\ 2 D. Eng., Prof., Dept. of Architecture and Architectural Engineering, Kyoto University \\ ohsaki@archi.kyoto-u.ac.jp
}

Editor's Note: The first author of this paper is one of the five winners of the 2020 Hangai Prize, awarded for outstanding papers that are submitted for presentation and publication at the annual IASS Symposium by younger members of the Association (under 30 years old). It is published here with permission of the editors of the proceedings of the IASS Symposium 2020/21 "Inspiring the Next Generation", that will be held in August 2021 in Guildford, UK.

DOI: https://doi.org/10.20898/j.iass.2020.010

\section{ABSTRACT}

This paper presents a design method of Auxetic Bending-Active Gridshells (ABAGs), which are curved surfaces generated from the initial flat grid with 2-dimensional auxetic patterns. One of the mechanical properties of ABAGs is that a dome-like shape of a curved surface can be easily obtained by bending a grid due to negative Poisson's ratio for in-plane deformation. Shapes of auxetic patterns are relevant to Poisson's ratio. Non-periodic and/or hybrid 2-dimensional auxetic patterns are developed for designing the initial flat grid of ABAGs. Shape parameters are the sizes of each plane unit for tuning its reentrant pattern, and two types of reentrant shapes are mixed on an initial flat grid. Using the non-uniform patterns, we can obtain an asymmetric and more complex free-form surface of ABAGs than those composed of a uniform reentrant pattern. Discrete Gaussian curvature at each node on a curved surface is computed for quantitatively evaluating the properties of shapes of the obtained surfaces. Possibility of ABAGs as a new design tool is demonstrated by showing that various shapes are generated through large deformation analysis with the forced displacements at the supports.

Keywords: bending-active structure, auxetic structure, gridshell, form-finding, discrete differential geometry

\section{INTRODUCTION}

Poisson's ratio is the ratio of the compressive strain to the tensile strain in the orthogonal direction in the uniaxial stress state. If the sign of Poisson's ratio is positive, a uniaxially compressed/stretched structure expands/shrinks in the transverse direction. By contrast, the structures with negative Poisson's ratio, which are called auxetic structures, have different mechanical properties than those with positive Poisson's ratio. A uniaxial compressed/stretched auxetic structure shrink/expand in the transverse direction [1-3].

Due to the negative Poisson's ratio for in-plane deformation, one of the structural properties of auxetic structures is that they can generate curved surfaces mainly with positive Gaussian curvature when subjected to out-of-plane bending deformation. This property of auxetic structures is well-known in the field of metamaterial design. On the other hand, elastic gridshell is one of the bendingactive structures, and its curved surface is composed of actively bent flexible beams from the initial flat grids [4-6]. Here, Auxetic Bending-Active Gridshell (ABAG) represents a bending-active gridshell, whose initial flat grid is designed as 2-dimensional auxetic pattern. Compared with the previous methods for designing a dome-like shape of an architectural roof, ABAG can be a useful tool because it enables us to generate easily free-form surface by elastically bending without assembly process, leading to reduction of construction time and cost.

Naboni et al. [7-9] carried out parametric study for designing $\mathrm{ABAGs}$ and analyzed them by using particle-spring method and finite element analysis. Moreover, they state in Ref. [7] that initial flat grids of ABAGs can be generated by using additive manufacturing technique, i.e., 3D printing. 


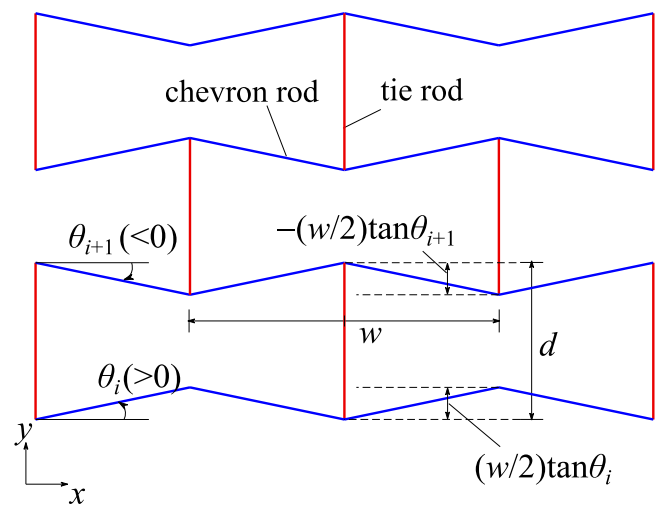

(a)

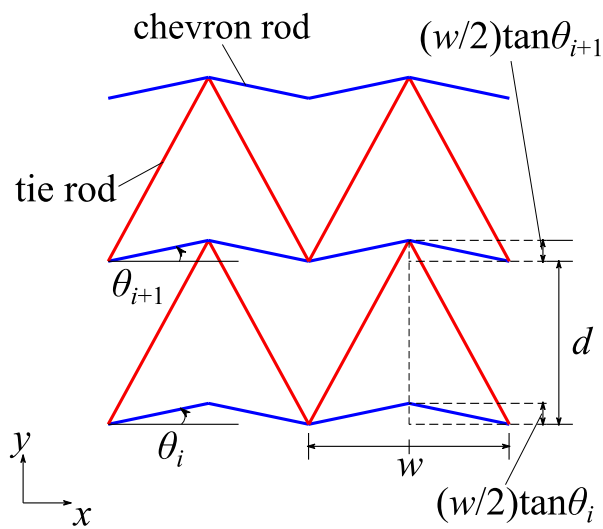

(b)

Figure 1: 2-dimensional reentrant units composed of chevron rods (blue line) and tie rods (red line) for realizing auxetic behaviors; (a) reentrant honeycomb, (b) reentrant triangle

Therefore, the connection between members is designed as rigid joint without any hinge joints.

La Magna and Knippers [10] proposed a method for arranging honeycomb patterns with gradually increasing Poisson's ratio from negative to positive on an initial flat plane using Schwartz-Christoffel mapping. The method in Ref. [10] enables us to obtain a curved surface which has Gaussian curvatures desired by designers. However, there have been still few researches addressing the design of ABAGs in the scale of architectural roof. Therefore, the further investigations of the structural properties of ABAGs are needed for its realization.

In this paper, we present a method for designing nonperiodic and/or hybrid ABAGs. 2-dimensional auxetic patterns are usually periodic because of their uniform reentrant shape. Reentrant honeycomb and reentrant triangle are the well-known 2-dimensional auxetic patterns and have also been used for design of various types of auxetic structures because of their structural simplicity and ease of arrangement. However, there is a limitation of obtained shapes of ABAGs generated from the conventional patterns.

We design non-periodic ABAGs composed of different patterns of reentrant honeycomb or reentrant triangle without periodicity. Hybrid ABAGs are also designed by mixing patterns of the two reentrant patterns. The two reentrant patterns are arranged in the direction perpendicular to the chevron rods, as illustrated in Fig. 1. Using nonuniform patterns, we can obtain an asymmetric and more complex free-form surfaces of ABAGs than those composed of a uniform reentrant pattern. Furthermore, we compute discrete Gaussian curvature at each node on a curved surface of ABAGs without using parametric forms of the surface [11]. The non-parametric curved shape can be easily discretized into a triangulated surface, and its property can be evaluated by the simple technique developed in the field of discrete differential geometry $[12,13]$. Finally, in the numerical examples, parametric study of large-deformation analysis is carried out to show that the proposed method can be regarded as a new design tool of ABAGs.

\section{2-DIMENSIONAL AUXETIC PATTERNS: REENTRANT HONEYCOMB ANDREENTRANT TRIANGLE}

A 2-dimensional auxetic structure, which has a negative Poisson's ratio for in-plane deformation, is composed of multiple periodic uniform patterns, which can completely fill a plane [14]. In this paper, we apply the reentrant honeycomb and reentrant triangle for designing an initial plane of ABAGs. Reentrant shapes consist of two types of beams: chevron rod (blue line) and tie rod (red line), as shown in Fig. 1. Let $i$ denote the number of chevron rods, and $\theta_{i}(i=1, \ldots, m), w$, and $d$ represent the angles between $x$-axis and the left end of the $i$ th chevron rod in the initial state, and the sizes along $x$ and $y$-axes of each unit, respectively. We define the angle so that anti-clockwise direction is positive for $\theta_{i}$. To avoid contacting or crossing of the members, the following constraints on reentrant honeycomb are to be satisfied: 


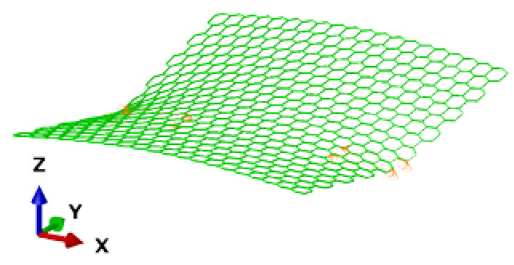

(a)

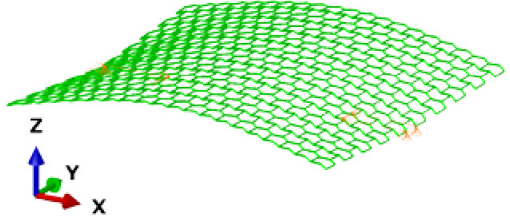

(b)

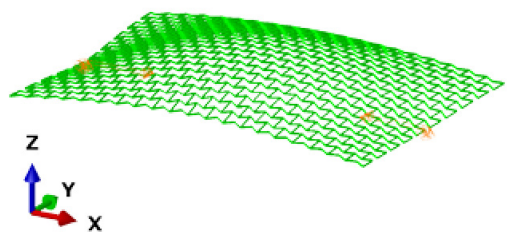

(c)

Figure 2: Comparison of relationships between Poisson's ratios and Gaussian curvatures; (a) $v>0$, (b) $v \approx 0$, (c) $v<0$

$$
\left\{\begin{array}{c}
d-\frac{1}{2} w\left(\tan \theta_{i}-\tan \theta_{i+1}\right)>0 \\
\quad \text { for } 0<\theta_{i}<\pi / 2 \text { and }-\pi / 2<\theta_{i+1}<0, \\
d-\frac{1}{2} w\left(-\tan \theta_{i}+\tan \theta_{i+1}\right)>0 \\
\text { for }-\pi / 2<\theta_{i}<0 \text { and } 0<\theta_{i+1}<\pi / 2 .
\end{array}\right.
$$

Similarly, the constraints on reentrant triangle are formulated as

$$
d-\frac{1}{2} w\left(\tan \theta_{i}-\tan \theta_{i+1}\right)>0
$$

Note that the term $\tan \theta_{i+1}$ in Eq. (2) is needed because the size $d$ represents the distance between the both ends of neighboring chevron rods.

Sign of the Gaussian curvature on a curved surface generated by bending the initial plane of $A B A G$ is dependent on the sign of Poisson's ratio for in-plane deformation [3]. Figure 2 shows the relationships between the obtained curved surfaces and Poisson's ratios. Poisson's ratios $v$ in Figs. 2 (a), (b), and (c) are positive, almost 0 , and negative, respectively. The obtained Gaussian curvatures on the corresponding surfaces are mainly negative, almost 0 , and positive, respectively.

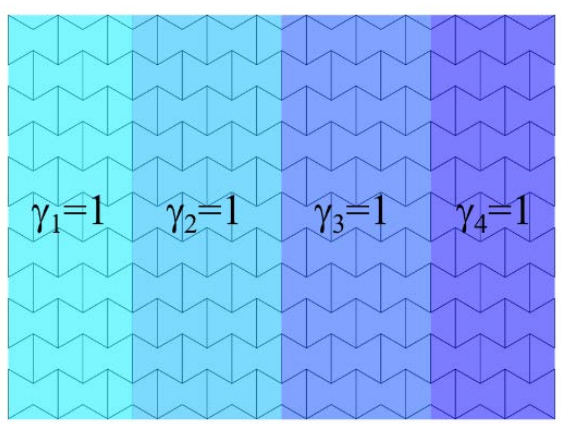

(a)

\section{NON-PERIODIC AND HYBRID ABAGS}

In this section, we propose a non-periodic and/or hybrid ABAGs. Simple uniform reentrant patterns are useful to design initial flat grids of ABAGs. However, uniform patterns can only generate simple and symmetric shapes of curved surface of ABAG. To overcome the limitation in the uniform reentrant units, we utilize various shapes and arrangements of reentrant units in an initial flat grid of ABAG. By sacrificing the simplicities of reentrant patterns, more complex shapes of curved surfaces are obtained.

\subsection{Non-periodic ABAGs}

We assign shape parameters $\gamma_{k}$ to an initial flat grid with $k$ sub-domains. The initial flat grid of a nonperiodic $\mathrm{ABAG}$ can be generated by tuning the size $w$ of each reentrant unit multiplied by shape parameters $\gamma_{k}$ without changing the lengths of tie rods and connectivity of nodes and rods. Figure 3 shows the initial flat planes with four sub-domains separated by the $y$-directional lines. Shape parameters $\gamma_{1}, \gamma_{2}, \gamma_{3}$, and $\gamma_{4}$ are assigned, respectively, in the corresponding sub-domains. In Fig. 3(a) and the numerical examples in Sec. 5, we set $\left|\theta_{i}\right|=\pi / 6$ ,$(i=1, \ldots, m)$ for reentrant patterns.

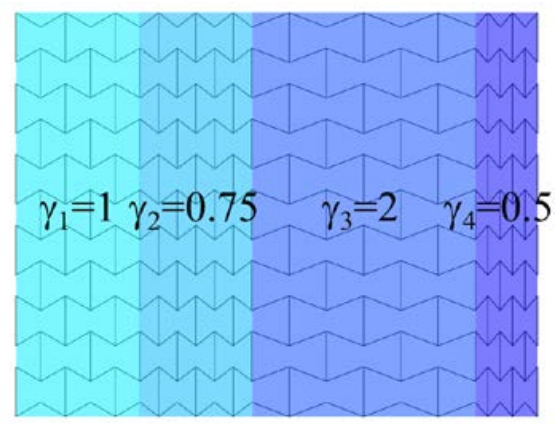

(b)

Figure 3: Tuning the size $w$ of reentrant units by parameter $\gamma_{k}(k=1, \ldots, 4)$; (a) periodic initial flat grid, (b) non-periodic initial flat grid 


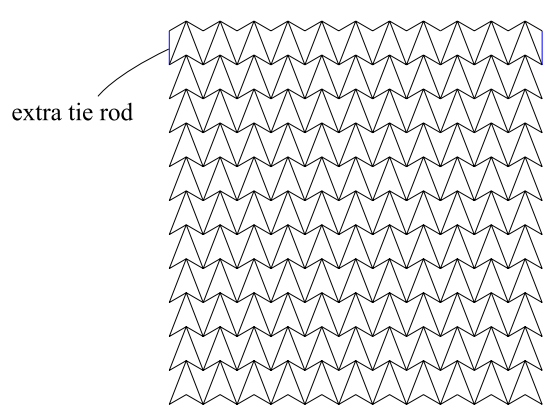

(a)

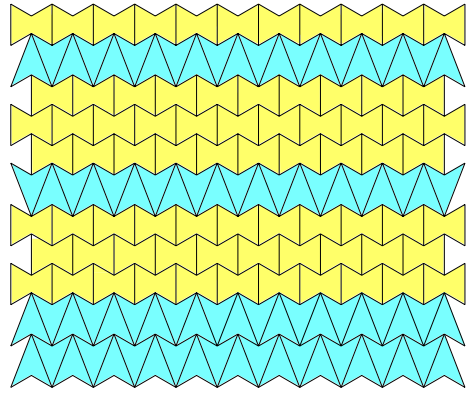

(b)

Figure 4: Initial flat grids of conventional and hybrid ABAGs; (a) conventional ABAG composed of uniform reentrant triangular units, (b) hybrid ABAG composed of reentrant honeycombs (yellow) and reentrant triangles (blue)

\subsection{Hybrid ABAGs}

A hybrid ABAG can have non-uniform Poisson's ratio of an initial flat grid, since it is composed of the two different reentrant units which have different Poisson's ratios. Initial flat grids of ABAGs are conventionally designed by using a single type of reentrant unit. We propose a simple method for designing initial flat grids filled with two different patterns in Fig. 1.

Each unit of the both reentrant patterns has two chevron rods. A reentrant triangle can be simply generated by arranging parallelly a uniform shape of chevron rod, while reentrant honeycomb is composed of two chevron rods which are symmetric with each other. The type of reentrant unit is defined based on the geometrical relationships of chevron rods. The signs of $\theta_{i}$ and $\theta_{i+1}$ define the geometrical relationship between the $i$ th and $(i+1)$ th chevron rods. If the product $\theta_{i} \theta_{i+1}$ is negative, then reentrant honeycombs are generated between the $i$ th and $(i+1)$ th chevron rods; otherwise, we obtain reentrant triangles between them. Figure 4(a) shows an initial flat grid composed of uniform reentrant triangles. Blue lines represent extra tie rods, which are assigned to the both ends of the $m$ th chevron rod so that it is not isolated. This procedure enables us to avoid the numerical difficulties during largedeformation analysis due to instability caused by isolated elements. Figure 4(b) shows a hybrid ABAG. Yellow and blue filled areas represent reentrant honeycomb and reentrant triangle, respectively.

\section{DISCRETE GAUSSIAN CURVATURE}

Discrete Gaussian curvature is defined for computing the curvature at nodes on a polyhedral discrete surface [12]. First, we discretize a curved surface of ABAG by triangulation. For simplicity, nodes are assumed to be located on the connections between chevron rods and tie rods. Then, we generate hexagonal cones at all nodes except those on boundaries and their neighboring nodes. Figure 5 shows triangular meshes on a curved surface. Circular and triangular marks represent the nodes on the connections and the additional nodes which are not on the connections, respectively. The position vectors of additional nodes $q_{2}, q_{3}, q_{5}$, and $q_{6}$ are defined as the average of the position vectors of their neighboring four nodes. For example, $q_{2}$ is defined by nodes $p, q_{1}, \mathrm{~B}$, and $\mathrm{D}$. The grey areas in Fig. 5 represent hexagonal meshes composed of six triangles on a curved surface.

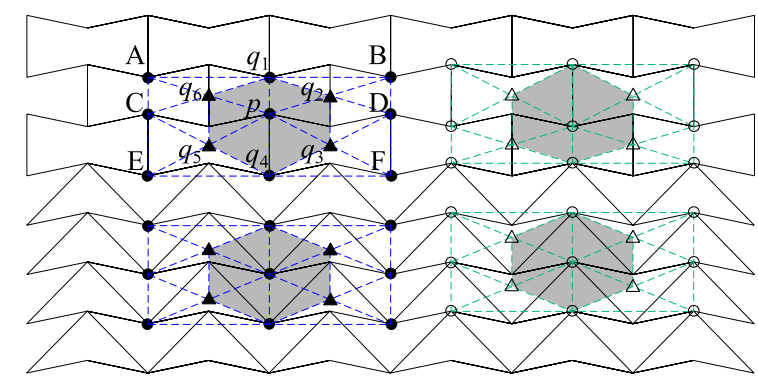

Figure 5: Hexagonal meshes composed of six triangles of each node on a curved surface of $A B A G$

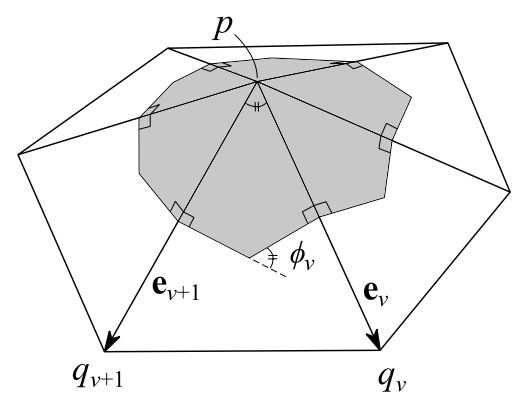

Figure 6: Hexagonal cone corresponding to node $p$, which is composed of six triangles defined with nodes $q_{v}(v=1$, ..., 6), and Voronoi region (grey) for computing angle defect 
Discrete Gaussian curvature at each node with six triangular meshes is formulated by angle defect from a flat plane. We use it as a measure for evaluating the amount of convexity and concavity of the generated curved surface. Figure 6 shows a hexagonal cone composed of nodes $p$ and $q_{v}(v=1, \ldots, 6)$. The grey area in Fig. 6 represents a Voronoi region generated by connecting the centers of the circumcircles surrounding triangles. Angle defect is defined as the difference between the sum of the outer angles $\phi_{v}$ and $2 \pi$. Note that the sum of $\phi_{v}$ is equal to the sum of inner angles of each triangular mesh on node $p$. Using the vector $\mathbf{e}_{v}$, which directs to node $q_{v}$ from $p$, the discrete Gaussian curvature $K_{p}$ is, therefore, computed as

$$
K_{p}=2 \pi-\sum_{v=1}^{6} \cos ^{-1}\left(\frac{\mathbf{e}_{v} \cdot \mathbf{e}_{v+1}}{\left|\mathbf{e}_{v}\right| \cdot\left|\mathbf{e}_{v+1}\right|}\right)
$$

For the cases of $K_{p}>0, K_{p}=0$, and $K_{p}<0$, we obtain a cone, a flat plane, or a saddle, respectively.

\section{NUMERICAL EXAMPLES}

In this section, we investigate the generated shapes of curved surfaces of non-periodic and/or hybrid ABAGs using discrete Gaussian curvature. Largedeformation analysis is carried out using Abaqus 2018 [15]. All numerical examples shown in this section have the same boundary conditions. Shape of the boundary of an ABAG is rectangle in the initial state of large-deformation analysis. The both ends of chevron rods are roller-supported, which can move along $x$-axis. All connections between tie rods and the first and the $m$ th chevron rods are also rollersupported, which can move on $x y$-plane. Figure 7 shows the process of large-deformation analysis for bending the initial flat grids. First, upward virtual load equivalent to self-load is applied to all members to avoid numerical difficulties due to a bifurcation buckling. Then, forced displacements along $x$-axis are given at the both ends of all chevron rods and the virtual vertical loads are released. In the following examples, we investigate the properties of ABAGs with five different patterns.

We use a material with Young's modulus $25 \mathrm{GPa}$ and Poisson's ratio 0.221. It is assumed to use glass fiber reinforced polymer (GFRP), which is a suitable material for bending-active structures because of its lightness, high strength, and flexibility $[4,5]$. The beam has a rectangle section with $0.01 \times 0.10(\mathrm{~m})$.

\subsection{Non-periodic ABAGs}

In this section, two curved surfaces of non-periodic ABAGs proposed in Sec. 3.1 are investigated. Surfaces 1 and 2 are composed of 242 reentrant honeycombs and 232 reentrant triangles, respectively. These reentrant units are tuned by shape parameters $\gamma_{k}(k=1, \ldots, 8)$ for the eight subdomains of each initial flat grid. Shape parameters $\gamma_{k}$ of Surfaces 1, 2, and 3 are defined, as shown in Table 1. Although Surface 3 is a non-periodic $\mathrm{ABAG}$, it is also classified into hybrid $\mathrm{ABAG}$ explained in Sec. 3.2. Therefore, the results of Surface 3 are shown in Sec. 5.3.

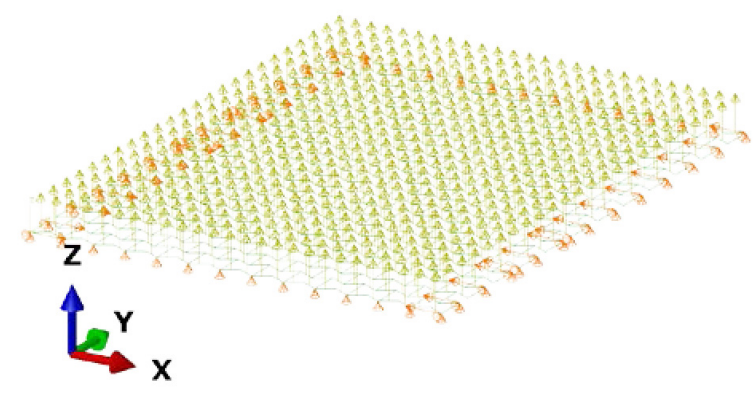

(a)

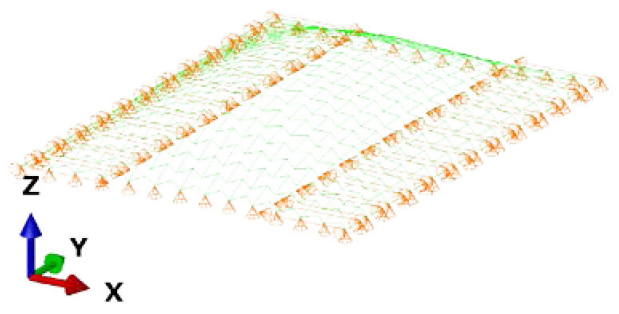

(b)

Figure 7: Loading conditions and forced displacements for a curved surface of periodic ABAG with reentrant honeycomb; (a) virtual upward load to all members, (b) giving forced-displacements at the ends of chevron rods

Table 1: Shape parameters $\gamma_{k}(k=1, \ldots, 8)$ assigned to the eight sub-domains along $y$-direction of initial flat grids of Surfaces 1, 2, and 3

\begin{tabular}{ccccccccc}
\hline & $\gamma_{1}$ & $\gamma_{2}$ & $\gamma_{3}$ & $\gamma_{4}$ & $\gamma_{5}$ & $\gamma_{6}$ & $\gamma_{7}$ & $\gamma_{8}$ \\
\hline Surface 1 & 1.00 & 0.50 & 1.20 & 0.80 & 0.50 & 1.00 & 0.50 & 0.80 \\
Surface 2 & 1.00 & 2.00 & 1.00 & 2.00 & 1.00 & 0.50 & 1.00 & 0.50 \\
Surface 3 & 1.00 & 0.75 & 1.50 & 0.50 & 0.75 & 1.25 & 0.50 & 1.00 \\
\hline
\end{tabular}




\section{Vol. 61 (2020) No. 4 December n. 206}

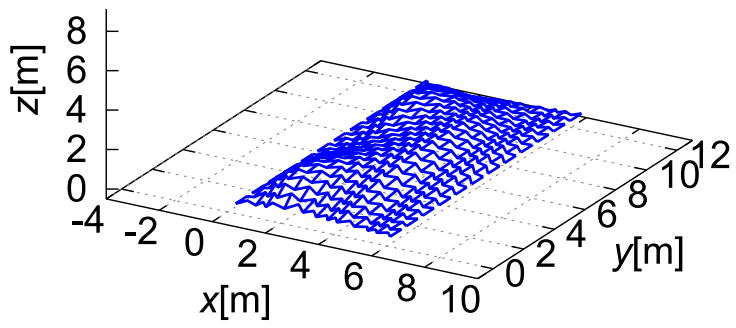

(a)

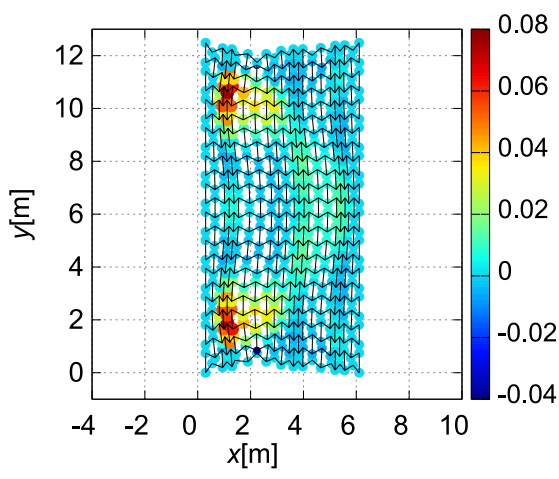

(b)

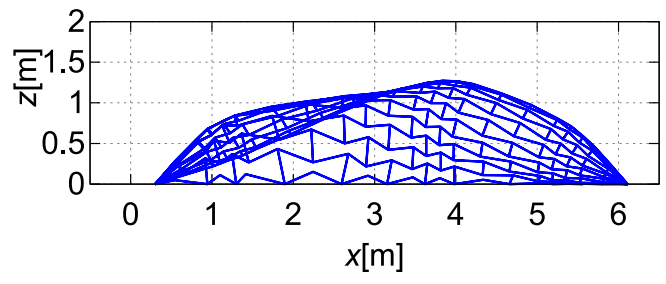

(c)

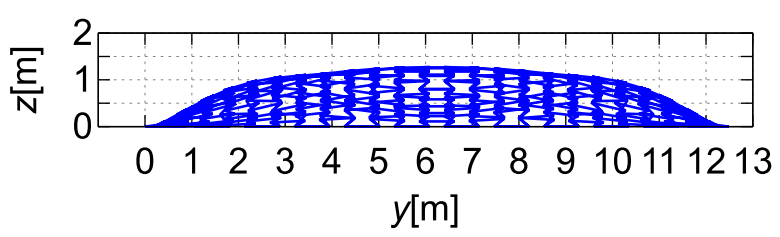

(d)

Figure 8: Surface 1, which is a non-periodic ABAG with 242 reentrant honeycombs; (a) curved surface, (b) xy-plane with the distribution of discrete Gaussian curvature, (c) xz-plane, (d) yz-plane

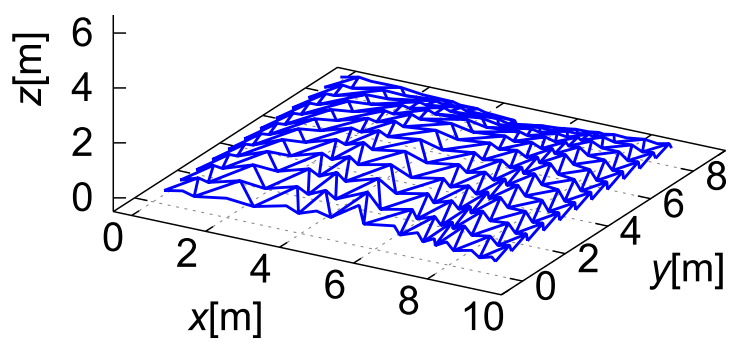

(a)

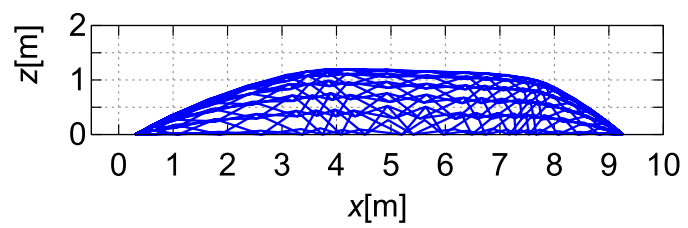

(c)

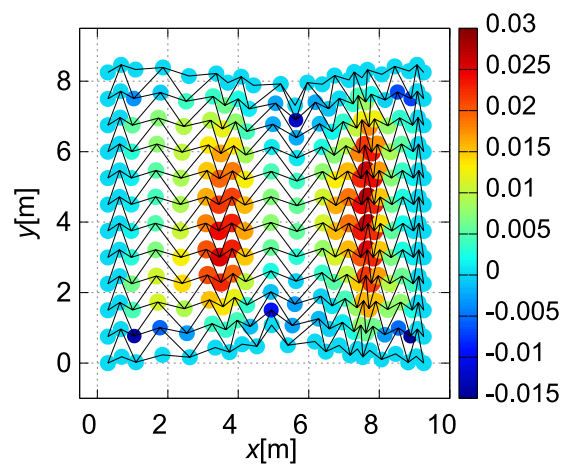

(b)

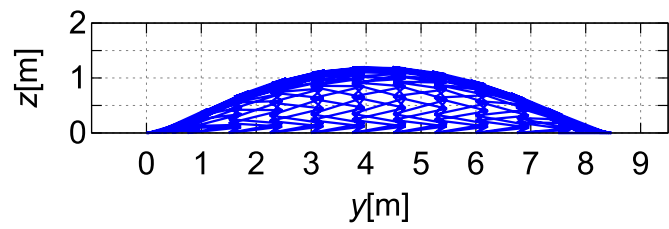

(d)

Figure 9: Surface 2, which is a non-periodic ABAG with 232 reentrant triangles; (a) curved surface, (b) xy-plane with the distribution of discrete Gaussian curvature, (c) xz-plane, (d) yz-plane

We set sizes of each unit of Surfaces 1 and 2 as $w=$ 0.75 and $d=0.75$. Forced displacements $0.30 \mathrm{~m}$ are given at the both ends of chevron rods along $x$-axis. The results of large-deformation analysis of Surfaces 1 and 2 are shown in Figs. 8 and 9, respectively. Figures $8(a),(b),(c)$, and (d) are a curved surface of
Surface 1, projection to $x y$-plane with a distribution of discrete Gaussian curvature at each node, projection to $x z$-plane, and projection to $y z$-plane, respectively. As shown in Fig. 8(b), larger values of discrete Gaussian curvature are obtained at nodes in the sub-domains to which smaller $\gamma_{k}$ are assigned. 
The largest values of discrete Gaussian curvature appear in the sub-domain assigned as $\gamma_{2}=0.50$. Distribution of discrete Gaussian curvature is symmetric with respect to the plane in $x$-direction. Asymmetric and symmetric shapes of Surface 1 are seen in the elevations Figs. 8(c) and (d). Figures 9(a), (b), (c), and (d) show the results of Surface 2. It is confirmed from Figure 9(b) that we can obtain larger values of discrete Gaussian curvature at nodes in sub-domains where assigned $\gamma_{k}$ are smaller, except boundaries.

\subsection{Hybrid ABAGs}

In this section, hybrid ABAGs are generated, and discrete Gaussian curvature is computed at each node on the curved surfaces. Surfaces 4 and 5 are composed of the both two reentrant patterns. Surface 4 has 145 reentrant honeycombs and 261 reentrant triangles. On the other hand, Surface 5 has 115 reentrant honeycombs and 252 reentrant triangles, which are arranged alternately. We set the sizes $w=$ 0.75 and $d=0.75$ for Surfaces 4 and 5. Forced displacements $0.30 \mathrm{~m}$ are given at the both ends of chevron rods. Figures 10 and 11 show the results of large-deformation analysis of Surfaces 4 and 5, respectively. Figure 10(a) shows the surface shape. Figure 10(b) is the projection to $x y$-plane with marks representing the values of discrete Gaussian curvature, which are symmetric with respect to a plane parallel with the $y$-axis, because the reentrant units are periodic in $x$-direction. Figures $10(\mathrm{c})$ and (d) show the projection to $x z$ - and $y z$-planes of Surface 4 . The results of Section 5 are shown in Fig. 11 in the same manner as Surface 4 in Fig. 10. Figure 11(b) shows the symmetric distribution of discrete Gaussian curvature of the surface of Surface 5, which is also composed of periodic reentrant units in $x$-direction. Furthermore, Surface 5 has the symmetry properties of reentrant units with respect to the two planes parallel to $x$ - and $y$-axes.

\subsection{Nonperiodic and hybrid ABAGs}

In this section, we investigate an example of the mixed non-periodic and hybrid ABAG. The mixed method is important because we can easily obtain more complex and asymmetric free-form surfaces of ABAGs than those in Secs. 5.1 and 5.2. We set the sizes $w=0.75$ and $d=0.75$ for the units. Forced displacements $0.30 \mathrm{~m}$ are given at both ends of chevron rods. Shape parameters $\gamma_{k}(k=1, \ldots, 8)$ are shown in Table 1. Surface 3 has 218 reentrant honeycombs and 232 reentrant triangles. Figure 12 shows the results of large-deformation analysis. Figures 12(a), (b), (c), and (d) are a curved surface of Surface 3, projection to $x y$-plane with a distribution of discrete Gaussian curvature at each node, projection to $x z$-plane, and projection to $y z$ plane, respectively. The distribution of discrete Gaussian curvature at each node is asymmetric; larger values of discrete Gaussian curvature are obtained at nodes in sub-domains whose shape parameters smaller than 1 .

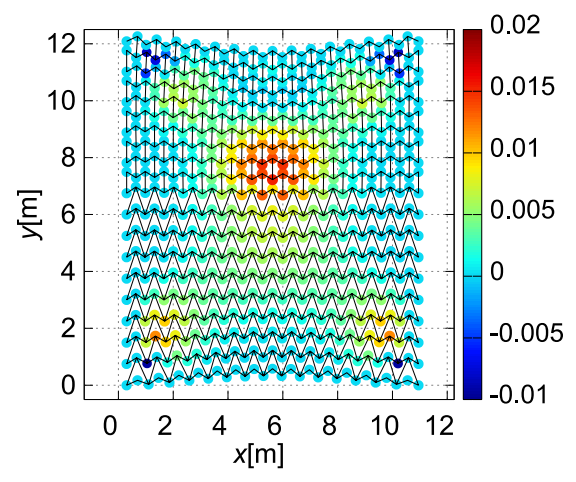

(b)

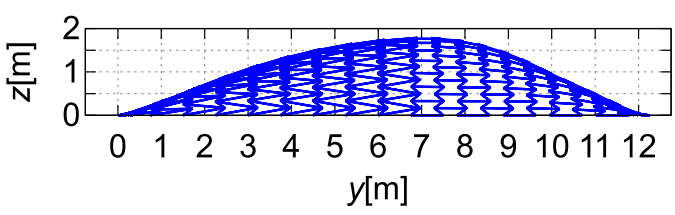

(d)

Figure 10: Surface 4, which is a hybrid ABAG with 145 reentrant honeycombs and 261 reentrant triangles; (a) curved surface, (b) xy-plane with the distribution of discrete Gaussian curvature, (c) xz-plane, (d) yz-plane 


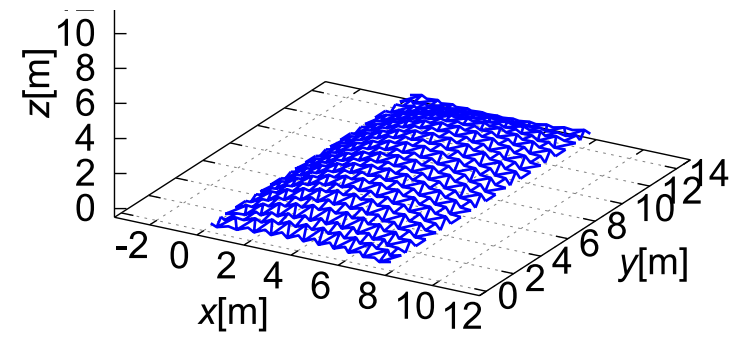

(a)

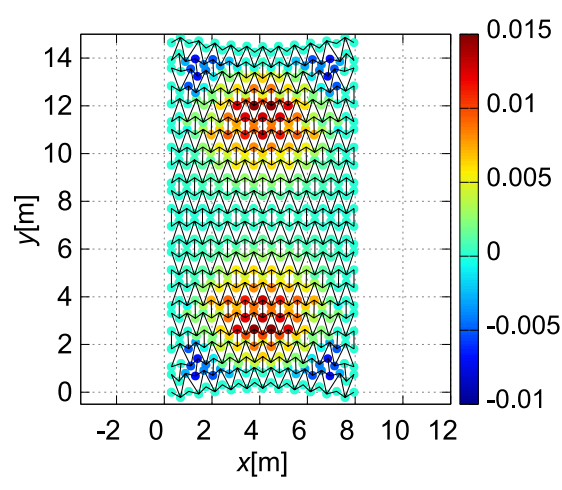

(b)

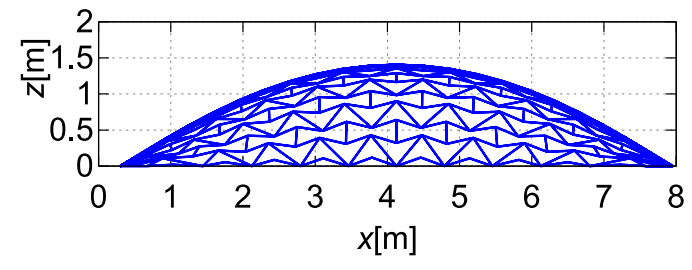

(c)

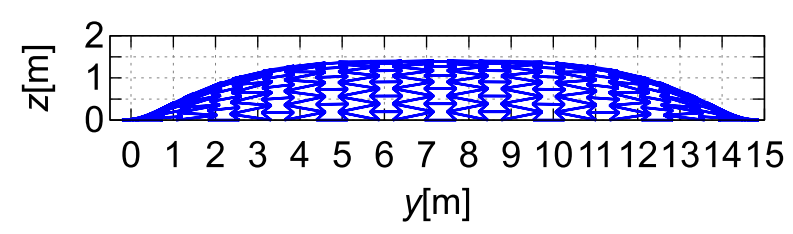

(d)

Figure 11: Surface 5, which is a hybrid ABAG with 115 reentrant honeycombs and 252 reentrant triangles; (a) curved surface, (b) xy-plane with the distribution of discrete Gaussian curvature, (c) xz-plane, (d) yz-plane

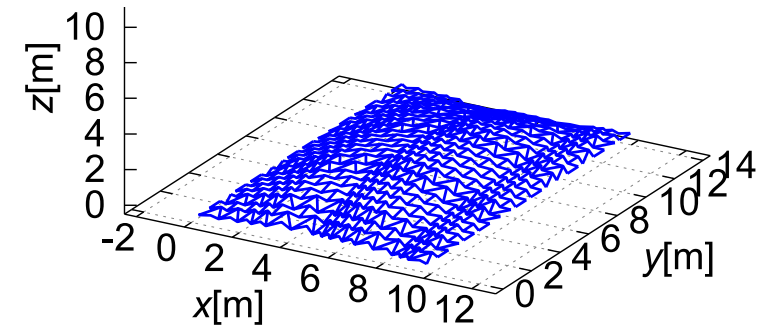

(a)

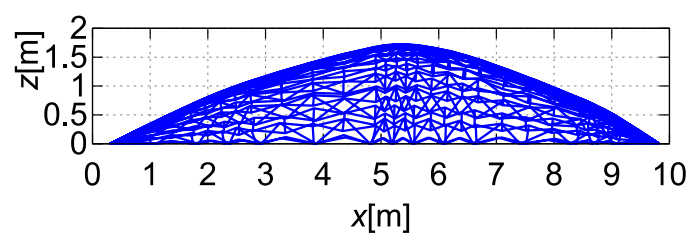

(c)

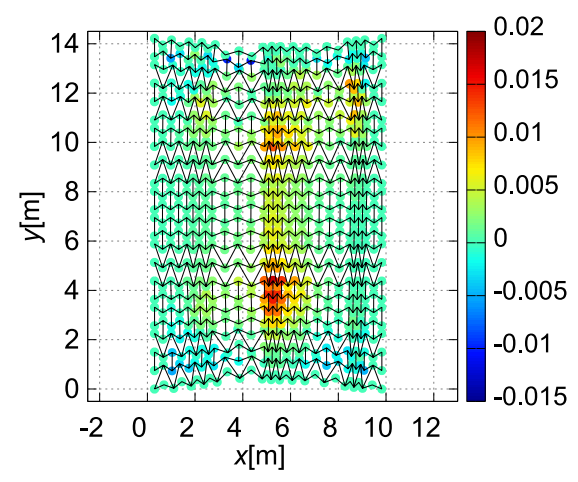

(b)

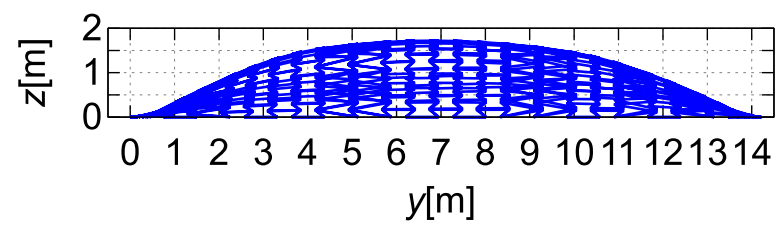

(d)

Figure 12: Surface 3, which is a non-periodic and hybrid ABAG with 218 reentrant honeycombs and 232 reentrant triangles; (a) curved surface, (b) xy-plane with the distribution of discrete Gaussian curvature, (c) xz-plane, (d) yz-plane

\section{CONCLUSIONS}

This paper presents a method for designing a new type of bending-active gridshell with negative Poisson's ratio for in-plane deformation, which is called non-periodic and/or hybrid ABAG. Using the proposed methods without any difficult design process of initial flat grids, we can obtain more complex shapes of curved surfaces than those composed of uniform reentrant units. We computed discrete Gaussian curvature for investigating the amount of convexity and concavity at each node on 
the obtained surfaces. Gaussian curvature can be used as a measure to avoid an unfavorable in view of structural or aesthetical properties. Since ABAG consists of discrete members, i.e., chevron rods and tie rods, it is natural to use discrete Gaussian curvature rather than continuous Gaussian curvature. Large-deformation analysis is carried out using Abaqus 2018 to generate various complex surfaces. Forced displacements are given at the supports actively to deform $\mathrm{ABAG}$ from a flat plane to a curved free-form surface.

Five parametric studies are conducted as the numerical examples. In all numerical examples, the obtained free-form surfaces of ABAGs show the auxetic behavior, which is confirmed by deformations projected to $x y$-plane. A non-periodic $\mathrm{ABAG}$ has various shape parameters $\gamma_{k}$ for reentrant units in the sub-domains. The obtained shape of a curved surface of non-periodic ABAG loses symmetry of discrete Gaussian curvature with respect to a plane parallel to $y$-axis. Moreover, we obtain larger values of discrete Gaussian curvature at nodes in sub-domains where smaller $\gamma_{k}$ are assigned. On the other hand, hybrid ABAG is composed of the both types of reentrant patterns, and various distributions of Poisson's ratio can be generated by arranging the sub-domains of different reentrant patterns. Therefore, a symmetric surface with respect to a plane parallel to $y$-axis is obtained. In the case of alternative arrangements of the two reentrant units, we can obtain a symmetric surface with respect to two planes parallel to $x$ - and $y$-axes. Compared with non-periodic ABAGs, hybrid ABAGs have the simple distributions of positive values of discrete Gaussian curvature. The reason for this property may be their simple arrangements of two different reentrant units as shown in Surfaces 4 and 5. The numerical examples showed that free-form surfaces of ABAGs are generated easily using the two proposed methods. The mixed method combining non-periodic and hybrid patterns was also investigated to show that an asymmetric free-form surface can be obtained. The distribution of discrete Gaussian curvature of Surface 3 clearly shows its complexity of a shape of a curved surface. The largest value of discrete Gaussian curvature of Surface 3 is obtained at a node in small $\gamma_{k}$ in the similar manner as non-periodic ABAGs.

This paper is a first attempt to analyze and evaluate the properties of ABAG utilizing discrete differential geometry. Furthermore, our proposed method can lead to future works of design of ABAG and its realization in an architectural scale.

\section{ACKNOWLEDGMENTS}

This work was supported by JSPS KAKENHI Number JP19J20405 and JST CREST Grant Number JPMJCR1911.

\section{DATA AVAILABILITY}

Some or all data, models, or codes that support the findings of this study are available from the corresponding author upon reasonable request.

\section{REFERENCES}

[1] R. Lakes, "Foam structures with a positive Poisson's ratio," Science, vol. 235, pp. 1038-1040, Feb. 1987. (DOI: 10.1126/science.235.4792.1038)

[2] K. E. Evans, "Auxetic polymers: a new range of materials," Endeavour, vol. 15, pp. 170-174, Feb. 1987. (DOI: 10.1016/01609327(91)90123-S)

[3] K. K. Saxena, R. Das and E. P. Calius, "Three decades of auxetics research - Materials with negative Poisson's ratio: A review," Advanced Engineering Materials, vol. 18, pp. 1847-1870, June 2016. (DOI: 10.1002/adem.201600053)

[4] C. Douthe, J. F. Caron and O. Baverel, "Gridshell structures in glass fibre reinforced polymers," Construction and Building Materials, vol. 24, pp. 1580-1589, Sept. 2010. (DOI: 10.1016/j.conbuildmat.2010.02.037)

[5] Y. Sakai and M. Ohsaki, "Discrete elastica for shape design of gridshells," Engineering Structures, vol. 169, pp. 55-67, Aug. 2018. (DOI: 10.1016/j.engstruct.2018.05.002)

[6] Y. Sakai, M. Ohsaki and S. Adriaenssens, "A 3-dimensional elastic beam model for formfinding of bending-active gridshells," International Journal of Solids and Structures, vol. 193-194, pp. 328-337, June 2020. (DOI: 10.1016/j.ijsolstr.2020.02.034)

[7] R. Naboni, S. Sartori and L. Mirante, "Adaptive-curvature structures with auxetic materials," Advanced Materials Research, vol. 1149, pp. 53-63, Aug. 2018. (DOI: 10.4028/www.scientific.net/AMR.1149.53) 
[8] R. Naboni and L. Mirante, "Computational design and simulation of bending-active auxetic structures," Gestão e Tecnologia de Projectos, vol. 11, pp. 59-71, Nov. 2016. (DOI: $10.11606 /$ gtp.v11i2.118141)

[9] R. Naboni and S. S. Pezzi, "Embedding auxetic properties in designing active-bending gridshells," in XX Congreso de la Sociedad Iberoamericana de Gráfica Digital 2016, São Paulo, Brazil, November, 9-11, 2016, Blucher, 2016. pp. 720-726. (DOI: 10.5151/despro-sigradi2016-490)

[10] R. La. Magna and J. Knippers, "Tailoring the bending behaviour of material patterns for the induction of double curvature," in Humanizing Digital Reality, K. De Rycke, Eds. Singapore: Springer, 2018, pp. 441-452. (DOI: 10.1007/978-981-10-6611-5_38)

[11] S. Fujita and M. Ohsaki, "Shape optimization of free-form shells using invariants of parametric surface," International Journal of Space Structures, vol. 25, pp. 143-157, Sept. 2010. (DOI: 10.1260/0266-3511.25.3.143)
[12] J. M. Sullivan, "Curvature measures for discrete surfaces," in Discrete Differential Geometry: An Applied Introduction, SIGGRAPH'05 2005, Los Angeles, USA, July 31, 2005, Chapter 3. (DOI: $10.1145 / 1198555.1198662)$

[13] M. Meyer, M. Desbrun, P. Schöder and A. H. Barr, "Discrete differential-geometry operators for triangulated 2-manifolds, " in Visualization and Mathematics III, H. C. Hege and K. Polthier, Eds. Berlin: Springer, 2003, pp. 35-57. (DOI: 10.1007/978-3-66205105-4_2)

[14] L. J. Gibson, M. F. Ashby, G. S. Schajer and C. I. Robertson, "The mechanics of twodimensional cellular materials," Proceedings of the Royal Society of London. Series A, Mathematical and Physical Sciences, vol. 382, pp. 25-42, July 2016. (DOI: 10.1098/rspa.1982.0087)

[15] Dassault Systèmes, "Abaqus 2018 User's manual," 2018. 